\title{
Universal Alcohol/Drug Screening in Prenatal Care: A Strategy for Reducing Racial Disparities? Questioning the Assumptions
}

\author{
Sarah C. M. Roberts • Amani Nuru-Jeter
}

Published online: 25 November 2010

(C) The Author(s) 2010. This article is published with open access at Springerlink.com

\begin{abstract}
Agencies and organizations promoting universal screening for alcohol and drug use in prenatal care argue that universal screening will reduce White versus Black racial disparities in reporting to Child Protective Services (CPS) at delivery. Yet, no published research has assessed the impact of universal screening on reporting disparities or explored plausible mechanisms. This review defines two potential mechanisms: Equitable Surveillance and Effective Treatment and identifies assumptions underlying each mechanism. It reviews published literature relating to each assumption. Research relating to assumptions underlying each mechanism is primarily inconclusive or contradictory. Thus, available research does not support the claim that universal screening for alcohol and drug use in prenatal care reduces racial disparities in CPS reporting at delivery. Reducing these reporting disparities requires more than universal screening.
\end{abstract}

Keywords Screening - Pregnancy $\cdot$ Substance-related disorders · Disparities - Child Welfare

\section{Introduction}

Government agencies and professional organizations encourage prenatal health care providers to implement universal screening for alcohol and drug use in prenatal

S. C. M. Roberts $(\bowtie)$

Alcohol Research Group, 6475 Christie Ave., Suite 400,

Emeryville, CA 94608, USA

e-mail: sroberts@arg.org

S. C. M. Roberts · A. Nuru-Jeter

School of Public Health, University of California, Berkeley, Berkeley, CA 94720, USA care [1-4]. Some agencies/organizations promoting universal screening argue that universal screening will reduce Black-White racial disparities in reporting to Child Protective Services (CPS) at delivery [5-7]. Importantly, no published research has assessed the impact of universal screening on CPS reporting disparities at delivery or explored the mechanisms through which universal screening could reduce these disparities. Understanding the impact of universal screening on CPS reporting disparities as well as the potential mechanisms through which universal screening could influence the disparities is essential as many providers begin to implement universal screening.

Understanding these possible mechanisms requires a look at screening in context of the larger system, which includes the policies and practices of providers, women, and institutions. Important points in this system are shown in Fig. 1.

Two potential mechanisms have been alluded to in discourse around universal screening. The first will be referred to as: Equitable Surveillance. The second will be referred to as: Effective Treatment.

Equitable Surveillance: Without explicitly stating the logic connecting universal screening to CPS reporting disparities, those arguing that universal screening will reduce disparities [5, 7] often cite the Pinellas County study [6]. In this study, Chasnoff et al. found that although White and Black pregnant women used alcohol and drugs at similar rates at their first prenatal care visit, Black women were 10 times more likely than White women to be reported to health authorities at delivery. In the discussion, the authors speculate that reporting disparities exist because prenatal providers primarily screen, and thus primarily identify, Black women. Chasnoff has since proposed universal screening as a solution to these reporting disparities [7]. The logic appears to be that universal 


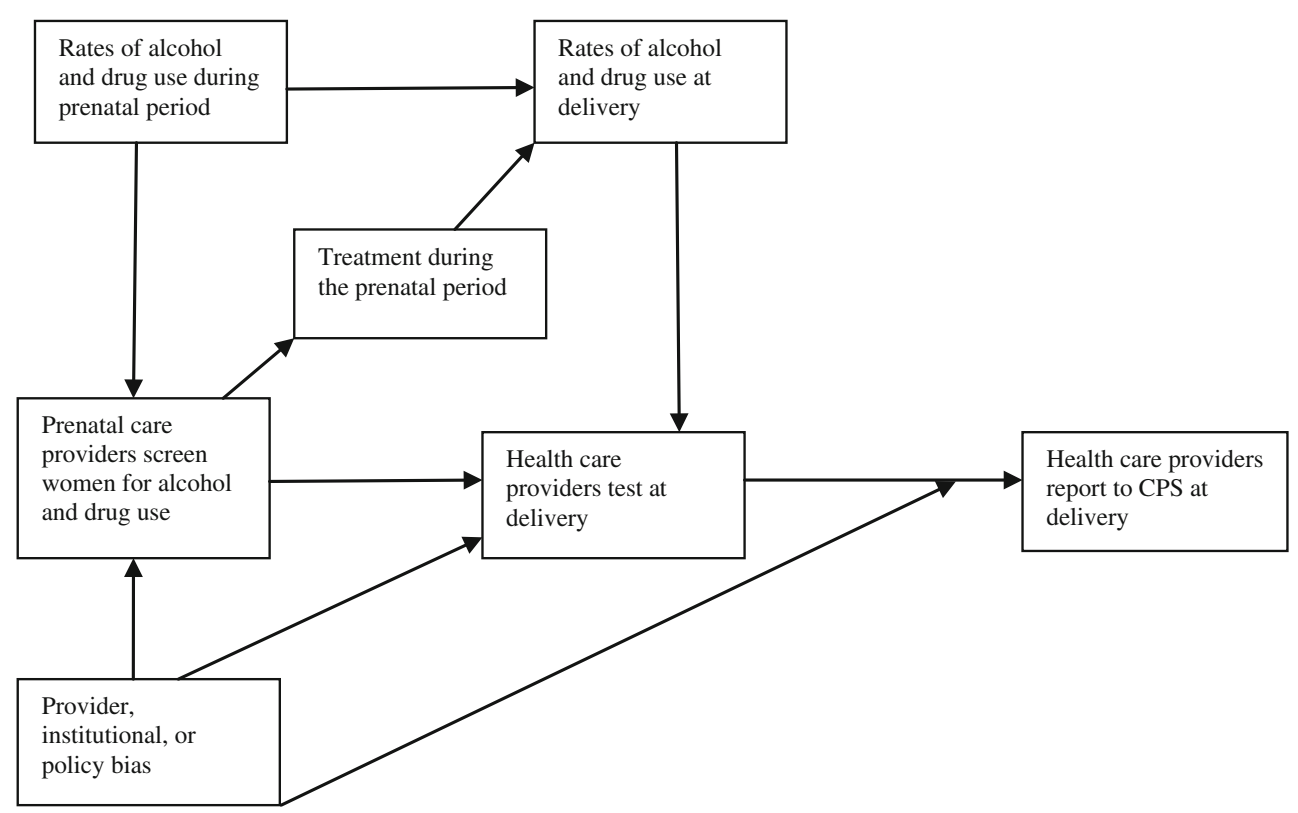

Fig. 1 Universal screening in prenatal care in context

screening will lead providers to identify more alcohol and drug use among White women during prenatal care. It follows that identifying more White women as using alcohol and drugs in prenatal care will lead providers to report more White women to CPS at delivery, thereby reducing reporting disparities. The argument is essentially that screening for alcohol and drug use in prenatal care functions as surveillance for CPS reporting at delivery. Thus, the goal of universal screening is to create more equitable surveillance where White women are just as likely to be screened and therefore reported as Black women.

Effective Treatment: Others suggest that universal screening helps identify pregnant women needing treatment earlier in pregnancy and ensures they receive needed treatment, either Brief Interventions (BI) or formal treatment $[5,8]$. Although unstated in the published literature, the premise underlying this argument is that providing effective treatment during the prenatal period reduces the need for and risk of CPS reporting at delivery. Reducing the number of women (including Black women) identified in prenatal care who continue to use alcohol and/or drugs through delivery will reduce the number of Black women reported at delivery, thereby reducing reporting disparities.

In addition to different perspectives on the role of screening in reducing reporting disparities, the assumptions underlying these two mechanisms differ. Equitable Surveillance assumes that: (1) prenatal providers screen fewer White than Black women; [6, 9] (2) White and Black women do not differ in patterns of alcohol and drug use during the prenatal period; [6] (3) rates of identification at delivery are disproportional to rates of use at delivery; and
(4) once providers identify pregnant women as using alcohol and/or drugs, they are equally likely to report White and Black women. Effective Treatment assumes that: (1) women identified through screening in prenatal care receive treatment; (2) there are no racial disparities in treatment receipt; (3) treatment provided to women identified through universal screening in prenatal care is effective; and (4) there are no racial disparities in treatment effectiveness.

\section{Methods}

This paper examines the plausibility of the Equitable Surveillance and Effective Treatment mechanisms by reviewing published literature related to each assumption outlined above. This review includes studies that relate to each of the specified assumptions relating to racial distribution of alcohol and drug use during pregnancy, screening for alcohol and drug use during prenatal care and at delivery, and brief interventions and treatment provided to women who have alcohol and/or drug use identified through universal screening in prenatal care. All included studies were published through June 2009 in Englishlanguage peer-reviewed journals. The one exception is the case of Equitable Surveillance Assumption 4, where disparities in a case that made it to the US Supreme Court are described. In addition to studies known to the authors through previous work on this subject, PubMed and Web of Science were searched to identify all additional relevant studies. Search terms included: "alcohol," "substance abuse," "cocaine," "methamphetamine," "marijuana," 
"heroin," “ecstasy," "illicit drugs," "pregnancy," "race," "screening," and "brief intervention." The resulting abstracts were examined to identify studies that addressed the assumptions outlined above. In addition to the keyword searches, we searched the reference sections of identified papers to find additional studies. We also used Web of Science and Google Scholar to find additional studies that had cited the studies we identified.

All studies we found that directly addressed the assumptions were included. For assumptions where few to no studies were identified, related studies were included and limitations to relevance described. The part of Equitable Surveillance Assumption 3 that relates to racial differences in use at delivery and in reduction and cessation over the course of pregnancy is the only part of this review in which there is a substantial body of research. For this portion of the review, studies that compared White and Black women directly with sufficient " $n$ " to detect differences, studies based in the United States, studies that measured alcohol use and drug use as separate variables, studies that were based on an entire population or subpopulation as well as prenatal clinic samples (including cohorts) were included. Studies where people were included or excluded based on potential confounders, such as those examining populations of women hospitalized for injuries during pregnancy and those excluding women with documented history of alcohol or drug abuse in medical charts, were excluded.

Given the limited research on the topic, and evidence available in non-peer reviewed outlets such as cases making it to the Supreme Court, we conducted as exhaustive a review as possible without restricting ourselves to specific inclusion/exclusion criteria. Therefore, this paper presents a nonsystematic, yet comprehensive review of the literature related to the assumptions underlying the Equitable Surveillance and Effective Treatment mechanisms outlined above.

\section{Findings}

Equitable Surveillance

\section{ES Assumption 1: Prenatal Providers Screen Fewer White than Black Women: INCONCLUSIVE}

No published studies directly address this question. We found two published studies relevant to whether providers screen fewer White than Black women during prenatal care. In a review of medical charts of women delivering at a university hospital, Kerker et al. [10] found that in some, but not all, prenatal clinics, prenatal care providers were less likely to document substance abuse history in charts of White versus Black women. In a study of provider beliefs about the prevalence of alcohol and drug use by race among adolescents in their care, Teagle and Brindis found that providers underestimated marijuana use among White and alcohol use among Black adolescents [11]. Assuming that provider beliefs about population distribution of alcohol and drug use predict which women they screen, providers would underscreen White women for marijuana and Black women for alcohol. In addition, some have suggested that public providers, who serve a higher proportion of Black women [12], are more likely to screen than private providers [6, 13]. However, while screening practices appear to vary across institutions [10, 14], it is not clear that public providers are more likely than private providers to screen $[1,15,16]$. More research is needed to better understand racial disparities in provider screening practices. Research related to Assumption1 is inconclusive.

ES Assumption 2: White and Black Women Do Not Differ in Patterns of Alcohol and Drug Use During the Prenatal Period: NOT SUPPORTED

Both White and Black women use alcohol and drugs during pregnancy. However, women differ with respect to both substance and pattern of use. Most [17-24], but not all [6, $25,26]$, studies have found that White women are more likely than Black women to use any alcohol during pregnancy. Some [20, 25, 27, 28], but not all [17, 24, 29], studies have found that racial differences disappear for binge and heavy alcohol use. Published research about whether White or Black women are more likely to use any drug during pregnancy is inconclusive [6, 19, 21, 23, 30]. However, studies consistently find White women more likely than Black women to use marijuana [6, 18, 30, 31] and, with one exception [18], less likely to use cocaine [6, 22, 30] and either less [21] or just as likely [18] to have heavier or more frequent drug use during pregnancy. In addition, one study found that while White women were more likely than Black women to use any alcohol, tobacco, or drugs during pregnancy, they were less likely than Black women to use two or three substances during pregnancy [32]. Therefore, it appears that White and Black women have similar rates of any use during the prenatal period. However, substances and possibly patterns differ. Further research is needed to better understand patterns of alcohol and drug use during pregnancy among White and Black women. Assumption 2 is not supported.

\section{ES Assumption 3: Rates of Identification at Delivery are Disproportional to Rates of Use at Delivery: NOT SUPPORTED}

Identification at delivery: Similar to prenatal care, some have suggested that racial disparities in urine toxicology 
testing at delivery are due to more testing in public than private hospitals $[13,33]$. Urine testing practices at delivery do appear to vary across hospitals [34]. However, variation is more complicated than differential testing in public versus private hospitals. A recent study found significant variation across hospitals in protocols providing guidance for identifying use at delivery [35]. Hospitals serving White and affluent patients had more detailed protocols specifying the need for maternal consent or discussion prior to testing. Hospitals serving more Black and lower-income women had less structured protocols [35]. Thus, providers at hospitals serving Black and low-income patients may have more discretion about who to test at delivery. There also may be differences within hospitals both with respect to insurance-type and race. A study based on interviews with nurse administrators at different hospitals found that some hospitals use insurance status (public insurance or self-pay) as a criterion to determine which women to test [36]. Kerker et al. [37] found that providers at a hospital serving low-income patients tested White women for cocaine at delivery less than they tested Black women. Similarly, Kunins et al. found that, among women delivering at an urban hospital, White and other women were less likely than Black women to be tested for drugs [38]. Thus, providers may test fewer White than Black women at delivery.

Rates of use at delivery: Kunins et al. also found that racial differences in testing at delivery were proportional to underlying differences in drug use at delivery [38]. A study by Vega et al. supports this finding, showing White women significantly less likely than Black women to have recently used either alcohol or drugs at delivery [39]. This finding differs from findings that measure alcohol and drug use at first prenatal visit [6], at any time since knowledge of pregnancy $[17,19-22,24,29]$, or as use within the past month [18, 25, 27, 28, 40], which find that White women are more likely or just as likely to use alcohol and drugs. Other, but not all [41], studies at delivery have found patterns similar to Vega and Kunins for drugs [42], especially cocaine [41, 43-48]. Findings about marijuana are mixed [44, 46, 49].

It is possible that there are racial differences in reduction or cessation of alcohol and drug use during pregnancy. In general, pregnant women are less likely than non-pregnant women to use alcohol and drugs (see $[18,19]$ ) and women tend to reduce use between the first and third trimesters $[24,30]$, although this may not apply to cocaine [30]. However, evidence is mixed as to whether White and Black women are similarly likely to reduce or cease alcohol use once pregnant. Some studies find that White and Black women are similarly likely to reduce or cease use once pregnant $[18,19,50,51]$, while other studies find that White women are more likely than Black women to reduce use $[25,52-54],{ }^{1}$ and one study found that White women are less likely than Black women to reduce use [55]. ${ }^{2}$ Evidence is more consistent for heavier drinkers. While White women are more likely than Black women to binge drink outside of pregnancy, White and Black women tend to have similar rates of binge drinking during pregnancy $[25,27,28,51]$. This suggests that among women who binge drink, White women may be more likely than Black women to stop during pregnancy. Additionally, one study found that among heavy drinkers, White and Black women were equally likely to quit, but White women were more likely to reduce their use [51]. Some studies find that White and Black women are equally likely to cease marijuana, cocaine, and drug use in general [18, 19]; however, other studies find that White women are more likely than Black women to cease use $[18,50,53]$ (See footnote 1). Therefore, it is possible that White women may be less likely than Black women to still be using drugs at delivery. Thus, part of what appears to be disproportionate testing at delivery may be testing proportionate to underlying differences in rates of use at delivery. Therefore, Assumption 3 is not supported.

\section{ES Assumption 4: Once Providers Identify Pregnant Women as Using Alcohol and/or Drugs, They are Equally Likely to Report White and Black Women: NOT SUPPORTED}

Findings from the Pinellas County study [6], described above, suggest that White women are less likely than Black women to be reported for alcohol and drug use at delivery. In the Pinellas County study, Chasnoff et al. found that White and Black women used alcohol and drugs at similar rates at their first prenatal visits, although White women were more likely than Black women to use marijuana and less likely to use cocaine. However, at delivery Black women were 10 times more likely than White women to be reported to health authorities [6].

Under a policy at the Medical University of South Carolina (MUSC) from 1989 to 1994 that was ruled unconstitutional by the US Supreme Court, prenatal providers informed pregnant women who tested positive for cocaine that they were required to get treatment, and if they missed a treatment appointment or had a second positive test, they would be reported to law enforcement and prosecuted [13, 56]. Forty-one women were prosecuted under this policy; 40 of the 41 were Black [57]. Other women, mostly White, tested positive for

\footnotetext{
${ }^{1}$ Hanna et al. (1994) does not report statistical significance levels, but the trends in this study with large sample size are in this direction.

2 Prager et al. (1984) does not report statistical significance levels, but the trend in this study with a large sample size is in this direction.
} 
methamphetamine and heroin, but were not reported to law enforcement [58]. Both the Pinellas County study and the MUSC prosecutions raise the possibility that disparities may reflect differential response to cocaine versus alcohol and other drugs [13].

While not directly about reporting disparities, studies about disparities in responses to CPS reports are consistent with studies about the initial reports. In a study of determinants of discharge custody decisions for a cohort of cocaine exposed newborns [59], Neuspiel et al. found that among women identified as using cocaine while pregnant, Black women were less likely than other women to have custody of their newborns at discharge. Similarly, MacMahon found that among women whose healthy babies tested positive for drugs, White women reported at delivery were less likely than Black women to never regain custody [60]. There does not appear to be research on disparities in reporting due to alcohol.

Research about reporting at delivery indicates that providers are less likely to report White than Black women. This appears due both to focus on cocaine and less reporting of White than Black women using the same substance. Assumption 4 is not supported.

\section{Effective Treatment}

\section{ET Assumption 1: Women Identified Through Universal Screening in Prenatal Care Receive Treatment: PARTIALLY SUPPORTED}

We found three studies that reported the percentage of women identified through universal screening in prenatal care who received either a BI or formal treatment. Kennedy et al. [61] found that $77 \%$ of women identified through universal screening in prenatal care in Massachusetts received a BI. Similarly, in Kaiser's Early Start program, about $70 \%$ of women who screened positive received at least one treatment visit [62]. Messer et al. [63] found that one-half (51\%) of low-income women identified through universal screening accepted treatment. Thus, while many who have alcohol and drug use identified through universal screening in prenatal care receive BIs or treatment, it appears that a large proportion of women identified through universal screening (23-49\%) receiving neither a BI nor more formal treatment. Assumption 1 is partially supported. However, research is needed to understand the consequences of screening for those who do not receive either a BI or treatment.

\section{ET Assumption 2: There are No Racial Disparities in Treatment Receipt: INCONCLUSIVE}

Kogan et al. [64] used the 1988 National Maternal and Infant Health Survey to examine racial differences in provider advice about alcohol and drug use during pregnancy. They found that White women were more likely than Black women to have received advice about alcohol and just as likely to have received advice about drugs [64]. In a study of low-income pregnant women who used alcohol, O'Connor and Whaley [65] found that White women were less likely than Black women to have received advice about alcohol use during pregnancy. Messer et al. [63] also examined a sample of low-income women and found White women less likely than Black women who needed treatment to accept treatment. The different findings suggest that racial differences in treatment receipt may vary by socioeconomic position. Evidence related to Assumption 2 is inconclusive.

\section{ET Assumption 3: Treatment Provided to Women Identified Through Universal Screening in Prenatal Care is Effective: INCONCLUSIVE}

There is limited research about the effectiveness of BIs and treatment provided to women identified through universal screening in prenatal care. In a systematic review, the United States Preventive Services Task Force (USPSTF) concluded that screening and BIs for alcohol are effective in general, but that studies during pregnancy were inconclusive [66]. A Cochrane review concluded that research about effectiveness of screening and BIs for alcohol for women in general was inconclusive [67]. Even less research has been conducted about effectiveness for drugs. The USPSTF concluded that it is not possible to determine from published research whether BIs and treatment provided to people whose drug use is identified through universal screening in primary care are effective, including during pregnancy [68]. Additional studies about effectiveness of screening and BIs during pregnancy have found mixed results, although more recent studies do seem to suggest that it may be effective [15, 69-72]. However, the Kaiser Permanente studies [15, 72] that include both alcohol and drugs focus only on birth outcomes. As the authors of these studies state, it is not known if the improvements in outcomes were attributable to women's reductions in use [72]. Thus, screening and BIs may be effective for alcohol, but the lack of research makes it impossible to determine for drugs. Assumption 3 is supported for alcohol use. Research relating to assumption 3 for drug use is inconclusive.

\section{ET Assumption 4: There are No Racial Disparities in Treatment Effectiveness: NO EVIDENCE}

There is no research about racial disparities in effectiveness of treatment provided to pregnant women as a result of identification through universal screening in prenatal care. Thus, Assumption 4 is not supported. 


\section{Conclusion}

Research relating to assumptions underlying both Equitable Surveillance and Effective Treatment is either inconclusive or contradictory. Available research does not support the claim that universal screening for alcohol and drug use in prenatal care will reduce racial disparities in CPS reporting at delivery. Research indicates that there are disparities at multiple points in this system, mainly in testing and reporting at delivery. These disparities may reflect more aggressive reporting of cocaine use [13] as well as less reporting of White than Black women who use the same substance. Research also suggests that a portion of what appears to be biases in responses may actually be proportional responses to underlying racial differences in rates of alcohol and drug use, especially at delivery. Thus, research contradicts most assumptions underlying Equitable Surveillance.

The source of reporting disparities does not appear to be bias in screening in prenatal care, but rather disparities in responses and solutions to pregnant women's alcohol and drug use. Logically, Effective Treatment makes more sense than Equitable Surveillance, because it accounts for the possibility that White women may be less likely than Black women to still be using drugs that put them at risk for CPS reporting at delivery. The main problem with Effective Treatment is the limited evidence that universal screening leads to treatment and that treatment provided as a result of identification in prenatal care is effective, especially for drugs. There has been no research to date on racial disparities in effectiveness of BIs and treatment provided as a result of universal screening in prenatal care. However, it seems unlikely that universal approaches such as universal screening and brief interventions will reduce disparities because reducing disparities generally requires specific attention to ensuring that interventions are effective for the group bearing disproportionate burden [73-75].

To make the Effective Treatment mechanism more likely to reduce racial disparities in reporting, researchers and practitioners need to develop, implement, and evaluate interventions and ensure that they meet the needs of Black women. Because screening for drug use in prenatal care may function as surveillance for women for whom interventions are ineffective (or not accepted), researchers and practitioners should be aware that efforts that identify women for whom the interventions are ineffective could place women under surveillance for CPS reporting. The perception that screening for drug use leads to CPS reporting is a reason that some women who use alcohol and drugs physically avoid and emotionally disengage from prenatal care [76] and, thus, is important to take into account. Developing and implementing such interventions that meet the needs of Black women will require a specific commitment on the part of policy-makers to reduce disparities [75]. It is unlikely to be accomplished as a side goal of universal screening.

Acknowledgments This project was supported by NIAAA Graduate Training on Alcohol Problems, T32 AA07240. Cheri Pies provided helpful comments on an earlier version of this paper.

Open Access This article is distributed under the terms of the Creative Commons Attribution Noncommercial License which permits any noncommercial use, distribution, and reproduction in any medium, provided the original author(s) and source are credited.

\section{References}

1. Littaua, R, Ramstrom, K, \& Jocson, M. (2006). Local MCAH jurisdiction survey on prenatal substance use screening data: Maternal, child and adolescent health/office of family planning branch, California Department of health services.

2. WSDOH. (2008). Washington state Department of health. Substance abuse during pregnancy: Guidelines for screening. Revised edition 2008.

3. ACOG. (2008). ACOG committee opinion No. 422: At-risk drinking and illicit drug use: Ethical issues in obstetric and gynecologic practice. Obstetrics and Gynecology, 112(6), 1449-1460.

4. Morse, B., Gehshan, S., \& Hutchins, E. (1997). Screening for substance abuse during pregnancy: Improving care, improving health. Arlington, VA: National Center for Education in Maternal and Child Health.

5. ACOG. (2004). ACOG committee opinion. Number 294, May 2004. At-risk drinking and illicit drug use: Ethical issues in obstetric and gynecologic practice. Obstetrics and Gynecology, 5(Pt 1), 1021-1031.

6. Chasnoff, I. J., Landress, H. J., \& Barrett, M. E. (1990). The prevalence of illicit-drug or alcohol use during pregnancy and discrepancies in mandatory reporting in Pinellas County, Florida. The New England Journal of Medicine, 322(17), 1202-1206.

7. Chasnoff IJ. (2007). Fetal alcohol spectrum disorders seminar for county MCAH teams and partners, 2007. Sacramento, CA.

8. Screening, brief intervention, and referral to treatment. (2008). Accessed 4 March 2008 at http://sbirt.samhsa.gov.

9. Weber, E. M. (2007). Child welfare interventions for drugdependent pregnant women: Limitations of a non-public health response. UMKC Law Review, 75(3), 789-845.

10. Kerker, B. D., Leventhal, J. M., Schlesinger, M., \& Horwitz, S. M. (2006). Racial and ethnic disparities in medical history taking: Detecting substance use among low-income pregnant women. Ethnicity and Disease, 16(1), 28-34.

11. Teagle, S. E., \& Brindis, C. D. (1998). Substance use among pregnant adolescents: A comparison of self-reported use and provider perception. The Journal of Adolescent Health, 22(3), 229-238.

12. IOM. (2002). Unequal treatment: Confronting racial and ethnic disparities in health care. Washington, DC: The National Academies Press.

13. Roberts, D. E. (1999). Killing the black body: Race, reproduction, and the meaning of liberty. New York: Vintage.

14. Herzig, K., Huynh, D., Gilbert, P., et al. (2006). Comparing prenatal providers' approaches to four different risks: Alcohol, tobacco, drugs, and domestic violence. Women and Health, 43(3), 83-101. 
15. Goler, N. C., Armstrong, M. A., Taillac, C. J., \& Osejo, V. M. (2008). Substance abuse treatment linked with prenatal visits improves perinatal outcomes: a new standard. Journal of Perinatology, 28(9), 597-603.

16. Gilbert, P., Herzig, K., Thakar, D., et al. (2007). How health care setting affects prenatal providers' risk reduction practices: A qualitative comparison of settings. Women and Health, 45(2), 41-57.

17. Caetano, R., Ramisetty-Mikler, S., Floyd, L. R., \& McGrath, C. (2006). The epidemiology of drinking among women of childbearing age. Alcoholism, Clinical and Experimental Research, 30(6), 1023-1030.

18. Muhuri, P. K., \& Gfroerer, J. C. (2009). Substance use among women: associations with pregnancy, parenting, and race/ethnicity. Maternal and Child Health Journal, 13(3), 376-385.

19. Johnson, S. F., McCarter, R. J., \& Ferencz, C. (1987). Changes in alcohol, cigarette, and recreational drug use during pregnancy: Implications for intervention. American Journal of Epidemiology, 126(4), 695-702.

20. Grant, T. M., Huggins, J. E., Sampson, P. D., Ernst, C. C., Barr, H. M., \& Streissguth, A. P. (2009). Alcohol use before and during pregnancy in western Washington, 1989-2004: Implications for the prevention of fetal alcohol spectrum disorders. American Journal of Obstetrics and Gynecology, 200(33), 278.e1-8.

21. Perreira, K. M., \& Cortes, K. E. (2006). Race/ethnicity and nativity differences in alcohol and tobacco use during pregnancy. American Journal of Public Health, 96(9), 1629-1636.

22. Streissguth, A. P., Grant, T. M., Barr, H. M., et al. (1991). Cocaine and the use of alcohol and other drugs during pregnancy. American Journal of Obstetrics and Gynecology, 164(5 Pt 1), $1239-1243$.

23. Wiemann, C. M., Berenson, A. B., \& San Miguel, V. V. (1994). Tobacco, alcohol and illicit drug use among pregnant women. Age and racial/ethnic differences. The Journal of Reproductive Medicine, 39(10), 769-776.

24. Ethen, M. K., Ramadhani, T. A., Scheuerle, A. E., et al. (2009). Alcohol consumption by women before and during pregnancy. Maternal and Child Health Journal, 13(2), 274-285.

25. Morris, D. S., Tenkku, L. E., Salas, J., Xaverius, P. K., \& Mengel, M. B. (2008). Exploring pregnancy-related changes in alcohol consumption between black and white women. Alcoholism, Clinical and Experimental Research, 32(3), 505-512.

26. Langlois, J. H., Ritter, J. M., \& Casey, R. J. (1991). Maternal and infant demographics and health status: A comparison of black, Caucasian, and Hispanic families. Journal of Biosocial Science, 23(1), 91-105.

27. Tsai, J., Floyd, R. L., Green, P. P., \& Boyle, C. A. (2007). Patterns and average volume of alcohol use among women of childbearing age. Maternal and Child Health Journal, 11(5), 437-445.

28. Ebrahim, S. H., Diekman, S. T., Floyd, R. L., \& Decoufle, P. (1999). Comparison of binge drinking among pregnant and nonpregnant women, United States, 1991-1995. American Journal of Obstetrics and Gynecology, 180(1 Pt 1), 1-7.

29. Flynn, H. A., Marcus, S. M., Barry, K. L., \& Blow, F. C. (2003). Rates and correlates of alcohol use among pregnant women in obstetrics clinics. Alcoholism, Clinical and Experimental Research, 27(1), 81-87.

30. Pegues, D. A., Engelgau, M. M., \& Woernle, C. H. (1994). Prevalence of illicit drugs detected in the urine of women of childbearing age in Alabama public health clinics. Public Health Report, 109(4), 530-538.

31. Abma, J. C., \& Mott, F. L. (1991). Substance use and prenatal care during pregnancy among young women. Family Planning Perspectives, 23(3), 117-122, 128.
32. Okah, F. A., Cai, J., \& Hoff, G. L. (2005). Term-gestation low birth weight and health-compromising behaviors during pregnancy. Obstetrics and Gynecology, 105(3), 543-550.

33. Gomez, L. E. (1997). Misconceiving mothers: Legislators, prosecutors, and the politics of prenatal drug exposure. Philadelphia: Temple University Press.

34. Oral, R., \& Strang, T. (2006). Neonatal illicit drug screening practices in Iowa: the impact of utilization of a structured screening protocol. Journal of Perinatology, 26(11), 660-666.

35. Zellman, G. L., Fair, C., Hoube, J., \& Wong, M. (2002). A search for guidance: Examining prenatal substance exposure protocols. Maternal and Child Health Journal, 6(3), 205-212.

36. Birchfield, M., Scully, J., \& Handler, A. (1995). Perinatal screening for illicit drugs: Policies in hospitals in a large metropolitan area. Journal of Perinatology, 15(3), 208-214.

37. Kerker, B. D., Horwitz, S. M., \& Leventhal, J. M. (2004). Patients' characteristics and providers' attitudes: Predictors of screening pregnant women for illicit substance use. Child Abuse and Neglect, 28(2), 209-223.

38. Kunins, H. V., Bellin, E., Chazotte, C., Du, E., \& Arnsten, J. H. (2007). The effect of race on provider decisions to test for illicit drug use in the peripartum setting. Journal of Womens Health (Larchmt), 16(2), 245-255.

39. Vega, W. A., Kolody, B., Hwang, J., \& Noble, A. (1993). Prevalence and magnitude of perinatal substance exposures in California. The New England Journal of Medicine, 329(12), $850-854$.

40. Havens, J. R., Simmons, L. A., Shannon, L. M., \& Hansen, W. F. (2009). Factors associated with substance use during pregnancy: Results from a national sample. Drug and Alcohol Dependence, 99(1-3), 89-95.

41. Vaughn, A. J., Carzoli, R. P., Sanchez-Ramos, L., Murphy, S., Khan, N., \& Chiu, T. (1993). Community-wide estimation of illicit drug use in delivering women: Prevalence, demographics, and associated risk factors. Obstetrics and Gynecology, 82(1), 92-96.

42. Yawn, B. P., Thompson, L. R., Lupo, V. R., Googins, M. K., \& Yawn, R. A. (1994). Prenatal drug use in Minneapolis-St Paul, Minn. A 4-year trend. Archives of Family Medicine, 3(6), 520-527.

43. Moser, J. M., Jones, V. H., \& Kuthy, M. L. (1993). Use of cocaine during the immediate prepartum period by childbearing women in Ohio. American Journal of Preventive Medicine, 9(2), $85-91$.

44. Cartwright, P. S., Schorge, J. O., \& McLaughlin, F. J. (1991). Epidemiologic characteristics of drug use during pregnancy: Experience in a Nashville hospital. The Southern Medical Journal, 84(7), 867-870.

45. Quinn, A. O., Van Mullem, C., Sturino, K., \& Broekhuizen, F. (1992). A multi-institutional analysis of perinatal cocaine use. Wisconsin Medical Journal, 91(6), 296-299.

46. Lake, M. F., Angel, J. L., Murphy, J. M., \& Poekert, G. (1992). Patterns of illicit drug use at the time of labor in a private and public hospital. Journal of Perinatology, 12(2), 134-136.

47. Statewide prevalence of illicit drug use by pregnant womenRhode Island. Mmwr. Morbidity and Mortality Weekly Report (1990) 39(14):225-227.

48. Gillogley, K. M., Evans, A. T., Hansen, R. L., Samuels, S. J., \& Batra, K. K. (1990). The perinatal impact of cocaine, amphetamine, and opiate use detected by universal intrapartum screening. American Journal of Obstetrics and Gynecology, 163(5 Pt 1), $1535-1542$.

49. MacGregor, S. N., Sciarra, J. C., Keith, L., \& Sciarra, J. J. (1990). Prevalence of marijuana use during pregnancy. A pilot study. The Journal of Reproductive Medicine, 35(12), 1147-1149. 
50. Harrison, P.A, \& Sidebottom, A.C. (2008). Alcohol and drug use before and during pregnancy: An examination of use patterns and predictors of cessation. Maternal and Child Health Journal, 13(3), 386-394.

51. Tenkku, L. E., Morris, D. S., Salas, J., \& Xaverius, P. K. (2009). Racial disparities in pregnancy-related drinking reduction. Maternal and Child Health Journal, 13(5), 604-613.

52. Ockene, J., Ma, Y., Zapka, J., Pbert, L., Valentine Goins, K., \& Stoddard, A. (2002). Spontaneous cessation of smoking and alcohol use among low-income pregnant women. American Journal of Preventive Medicine, 23(3), 150-159.

53. Hanna, E. Z., Faden, V. B., \& Dufour, M. C. (1994). The motivational correlates of drinking, smoking, and illicit drug use during pregnancy. Journal Of Substance Abuse, 6(2), 155-167.

54. Stephens, C. J. (1985). Alcohol consumption during pregnancy among Southern city women. Drug and Alcohol Dependence, 16(1), 19-29.

55. Prager, K., Malin, H., Spiegler, D., Van Natta, P., \& Placek, P. J. (1984). Smoking and drinking behavior before and during pregnancy of married mothers of live-born infants and stillborn infants. Public Health Report, 99(2), 117-127.

56. United States Supreme Court. Ferguson v. City of Charleston. Supreme Court Reporter; 2001; 121:1281.

57. Lester, B. M., Andreozzi, L., \& Appiah, L. (2004). Substance use during pregnancy: Time for policy to catch up with research. Harm Reduction Journal, 1(1), 5.

58. Gostin, L. O. (2001). The rights of pregnant women: The Supreme Court and drug testing. The Hastings Center Report, 31(5), 8-9.

59. Neuspiel, D. R., Zingman, T. M., Templeton, V. H., DiStabile, P., \& Drucker, E. (1993). Custody of cocaine-exposed newborns: Determinants of discharge decisions. American Journal of Public Health, 83(12), 1726-1729.

60. MacMahon, J. R. (1997). Perinatal substance abuse: The impact of reporting infants to child protective services. Pediatrics, $100(5), \mathrm{e} 1$.

61. Kennedy, C., Finkelstein, N., Hutchins, E., \& Mahoney, J. (2004). Improving screening for alcohol use during pregnancy: The Massachusetts ASAP program. Maternal and Child Health Journal, 8(3), 137-147.

62. Taillac, C., Goler, N., Armstrong, M.A., Haley, K., Osejo, V. (2007). Early Start: An Integrated Model of Substance Abuse Intervention for Pregnant Women. The Permanente Journal 11(3).

63. Messer, K., Clark, K. A., \& Martin, S. L. (1996). Characteristics associated with pregnant women's utilization of substance abuse treatment services. American Journal of Drug and Alcohol Abuse, 22(3), 403-422.
64. Kogan, M. D., Kotelchuck, M., Alexander, G. R., \& Johnson, W. E. (1994). Racial disparities in reported prenatal care advice from health care providers. American Journal of Public Health, 84(1), $82-88$.

65. O'Connor, M. J., \& Whaley, S. E. (2006). Health care provider advice and risk factors associated with alcohol consumption following pregnancy recognition. Journal of studies on alcohol, 67(1), 22-31.

66. Whitlock, E. P., Green, C. A., \& Polen, M. R. (2004). Behavioral counseling interventions in primary care to reduce risky/harmful alcohol use. Portland: AHRQ.

67. Kaner, E. F., Dickinson, H. O., Beyer, F., et al. (2009). The effectiveness of brief alcohol interventions in primary care settings: A systematic review. Drug and Alcohol Review, 28(3), 301-323.

68. Polen, M., Whitlock, E., Wisdom, J., Nygren, P., \& Bougatsos, C. (2008). Screening in primary care settings for illicit drug use staged systematic review for the United States preventive services task force: Agency for healthcare research and quality. Rockville, MD: AHRQ Publication No. 08-05108-EF-s.

69. Chang, G., McNamara, T. K., Orav, E. J., et al. (2005). Brief intervention for prenatal alcohol use: A randomized trial. Obstetrics and Gynecology, 105(5 Pt 1), 991-998.

70. Chang, G., Wilkins-Haug, L., Berman, S., \& Goetz, M. A. (1999). Brief intervention for alcohol use in pregnancy: A randomized trial. Addiction, 94(10), 1499-1508.

71. O'Connor, M. J., \& Whaley, S. E. (2007). Brief intervention for alcohol use by pregnant women. American Journal of Public Health, 97(2), 252-258.

72. Armstrong, M. A., Gonzales Osejo, V., Lieberman, L., Carpenter, D. M., Pantoja, P. M., \& Escobar, G. J. (2003). Perinatal substance abuse intervention in obstetric clinics decreases adverse neonatal outcomes. Journal of Perinatology, 23(1), 3-9.

73. Marmot, M., Bobak, M., \& Davey Smith, G. (1995). Explanations for social inequalities in health. In B. Amick III, S. Levine, A. Tarlov, \& D. Chapman Walsh (Eds.), Society and health (pp. 172-210). New York: Oxford University Press.

74. Frohlich, K. L., \& Potvin, L. (2008). Transcending the known in public health practice: the inequality paradox: The population approach and vulnerable populations. American Journal of Public Health, 98(2), 216-221.

75. Keppel, K., Bilheimer, L., \& Gurley, L. (2007). Improving population health and reducing health care disparities. Health Affairs (Millwood), 26(5), 1281-1292.

76. Roberts, S. C. M., \& Nuru-Jeter, A. (2010). Women's perspectives on screening for alcohol and drug use in prenatal care. Women's Health Issues, 20(3), 193-200. 